\title{
The Effect of Lipopolysaccharide Pretreatment on Trauma-Induced Dark Neurons in the Hippocampus
}

\author{
Mansoureh Eslami $^{1 *}$, Leila Alizade ${ }^{2}$, Mohammad Sayyah ${ }^{3 *}$ \\ ${ }^{1}$ Faculty of Paramedical Sciences, Shahid Beheshti University of Medical Sciences, Tehran, Iran \\ ${ }^{2}$ Shefa Neuroscience Research Center, Khatam Alanbia Hospital, Tehran, Iran \\ ${ }^{3}$ Department of Physiology and Pharmacology, Pasteur Institute of Iran, Tehran, Iran
}

Article Info:

\section{A BSTRACT}

Introduction: Traumatic brain injury (TBI) is a serious medical condition that affects annually year more than 2 million people worldwide. This study was aimed to evaluate the effect of lipopolysaccharide (LPS) on the number of dark neurons induced by TBI in the parieto-temporal cortex. Materials and Methods: LPS $(0.1$ and $0.5 \mathrm{mg} / \mathrm{kg})$ was injected i.p. to rats 5 days before induction of TBI in the parieto-temporal cortex. Using toluidine blue staining, we evaluated the number of dark neurons in the hippocampus in sham and LPS preconditioned rats 4 and 12 hours after the trauma. Results: The increase in dark neurons was significantly higher after $4 \mathrm{~h}$ of trauma compared to $12 \mathrm{~h}$ after TBI. LPS preconditioning significantly decreased the mean number of dark neurons in the hippocampus. In LPS preconditioned rats the mean number of dark neurons was also significantly higher 4 hours after trauma compared to $12 \mathrm{~h}$ after TBI. Conclusion: These data indicate that hippocampal cellular damage produced during the first hours after induction of TBI. This cellular damage could be prevented by administration of LPS.

Key words:

1. Neurons

2. Hippocampus

3. Rats

*Corresponding Author: Mansoureh Eslami, Mohammad Sayyah

E-mail:eslami342@sbmu.ac.ir,sayyahm2@pasteur.ac.ir 


\title{
اثر ييش درمانى با لييو يلىساكاريد بر نورونهاى تيرهُ ناشى از تروما در هييوكامٍ
}

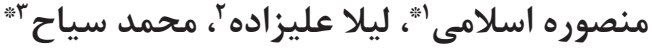 \\ 'دانشكده يِيرايزشكى، دانشعاه علوم يزشكى شهيد بهشتى، تهران، ايران

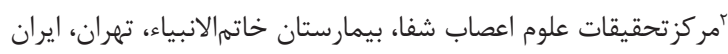

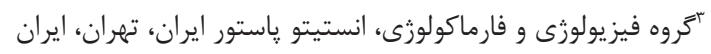

اطلاعات مقاله:

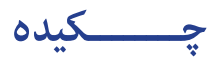

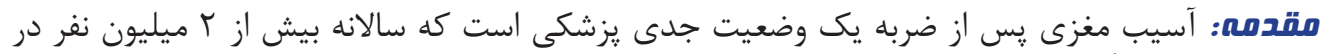

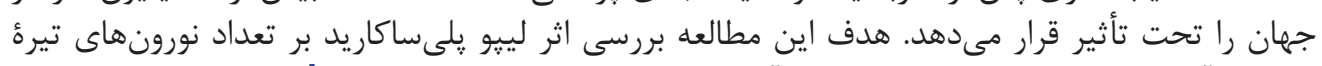

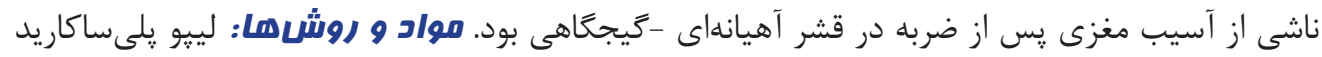

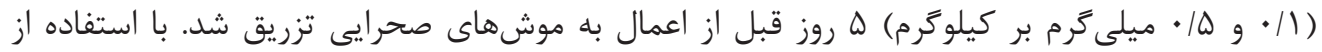

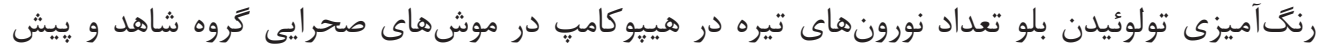

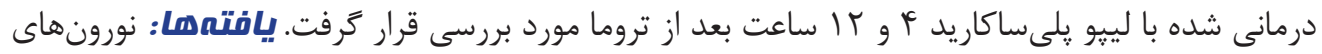

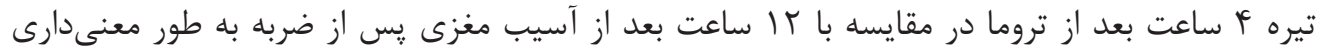

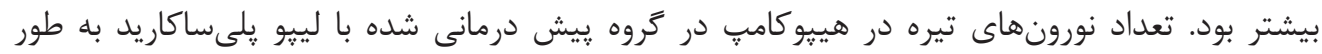

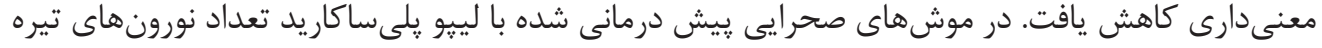

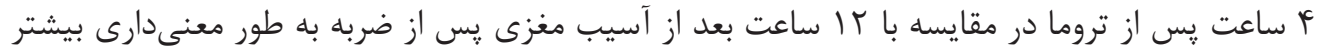

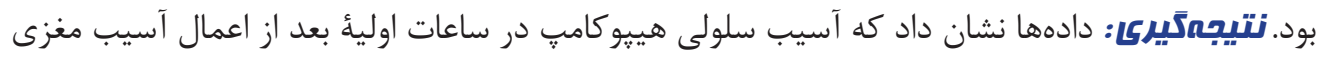

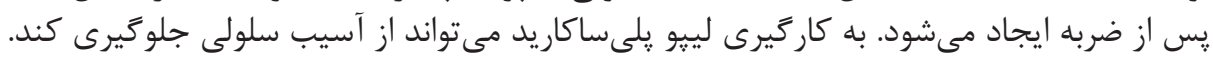

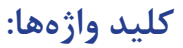

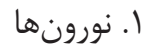

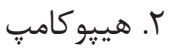

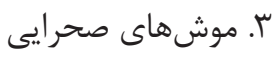


مواد و روشها - - - مان

حيوانات

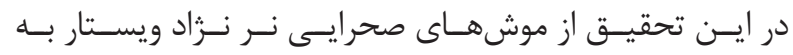

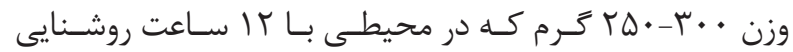

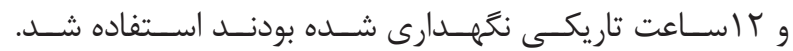

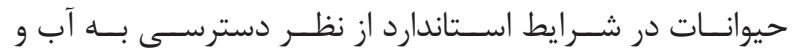

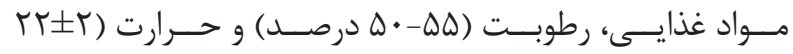

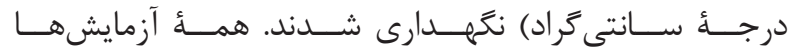

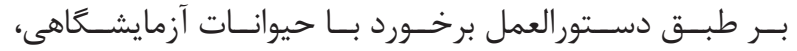

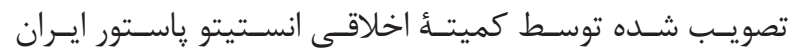

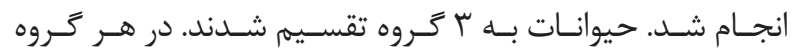

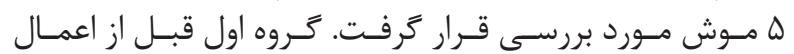

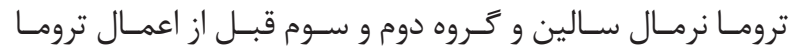

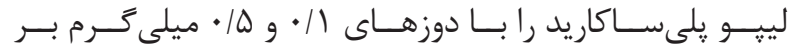

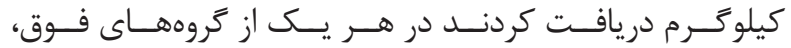

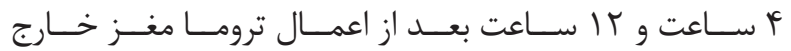

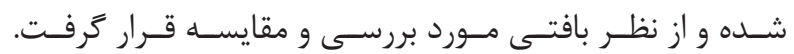

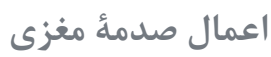

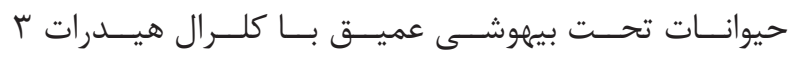

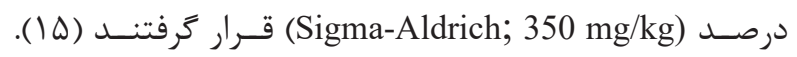

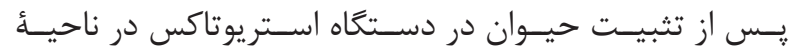

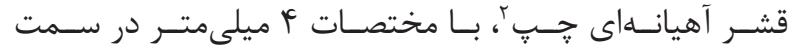

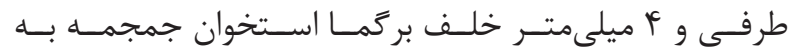

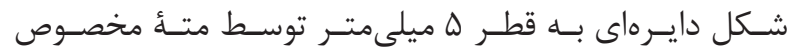

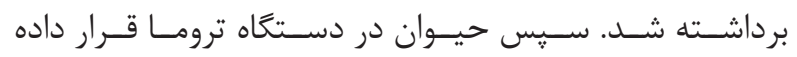

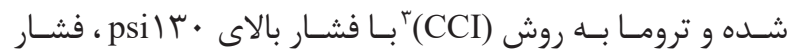

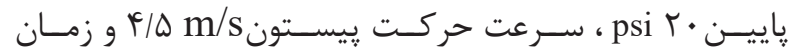

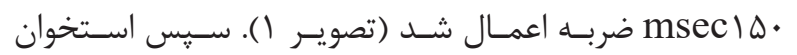

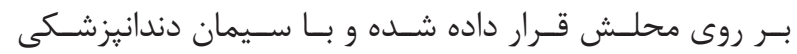

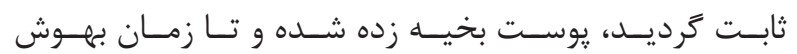

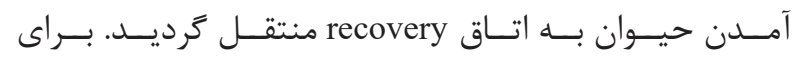

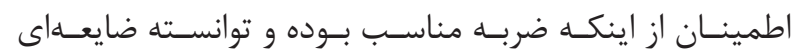

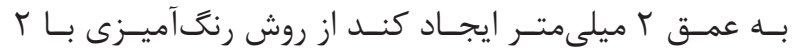

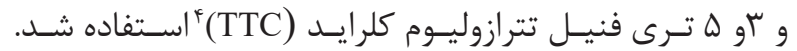

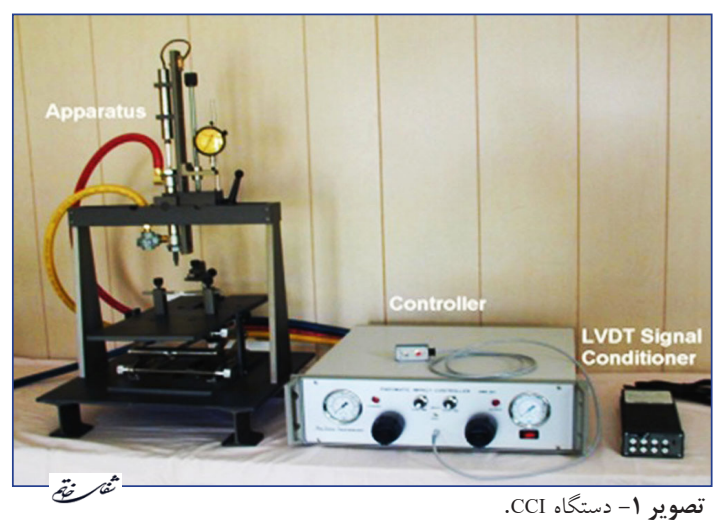

${ }^{1}$ Traumatic brain injury

${ }^{2}$ Left parietal cortex

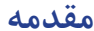

آسـيبهاى ناشـى از ضربــات مغـزى (TBI) ' يكى از مشـكلات

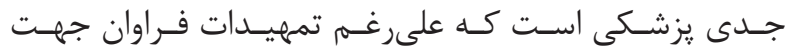

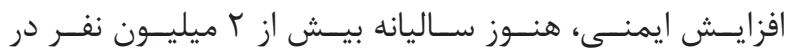

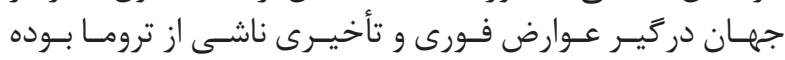

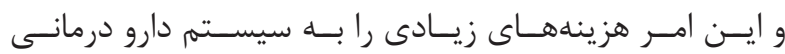

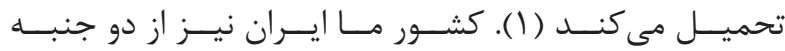

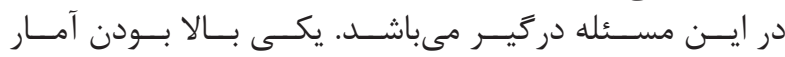

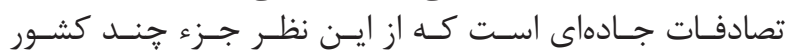

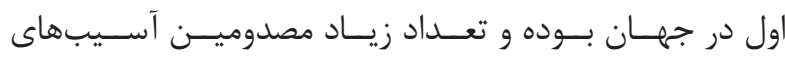

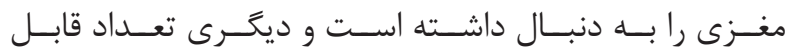

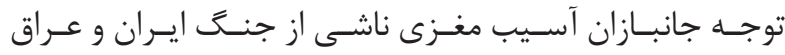

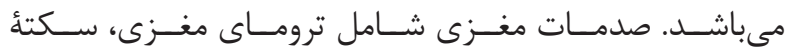

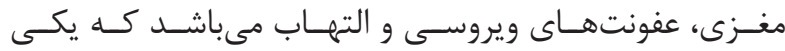

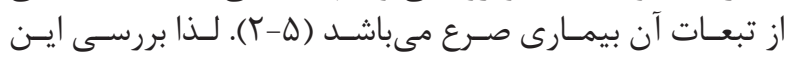

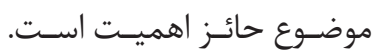

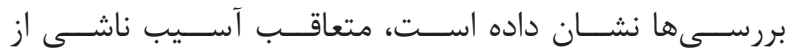

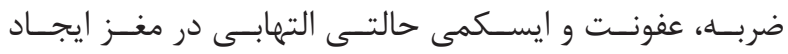

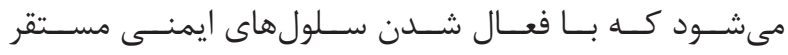

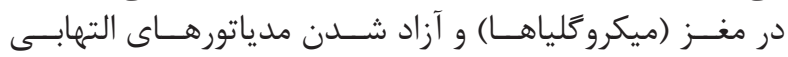

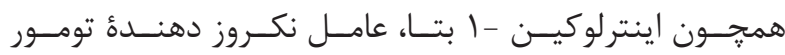

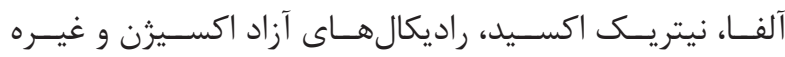

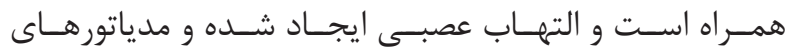

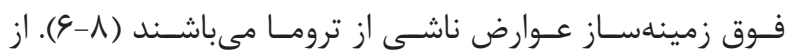

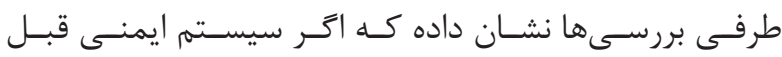

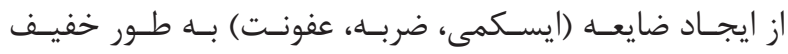

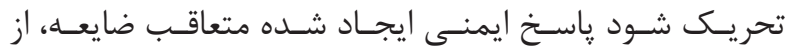

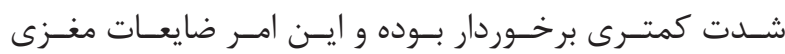

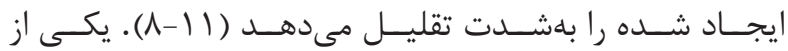

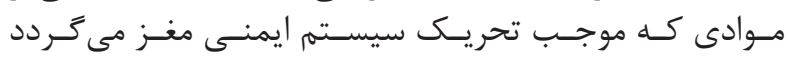

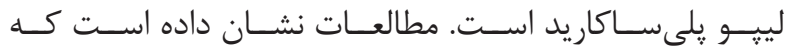

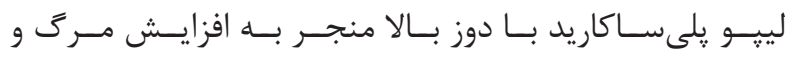

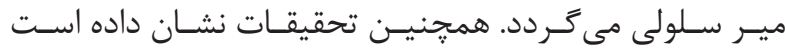

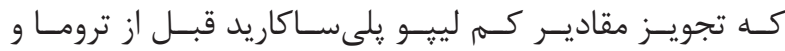

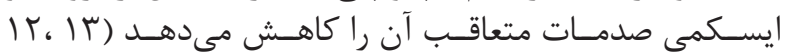

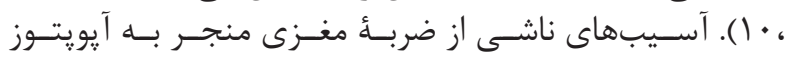

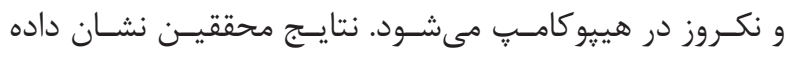

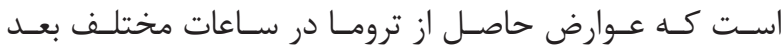

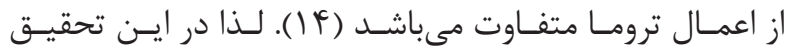

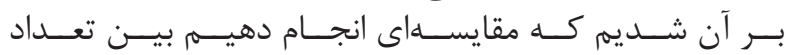

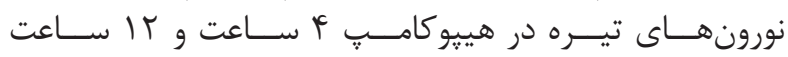

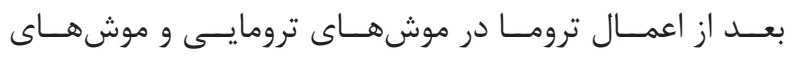

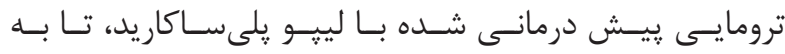

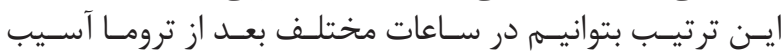

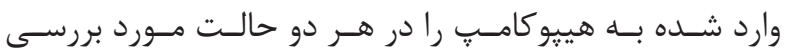

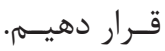

${ }^{3}$ Controlled cortical injury

${ }^{4}$ Triphenyl tetrazolium chloride 2,3,5 


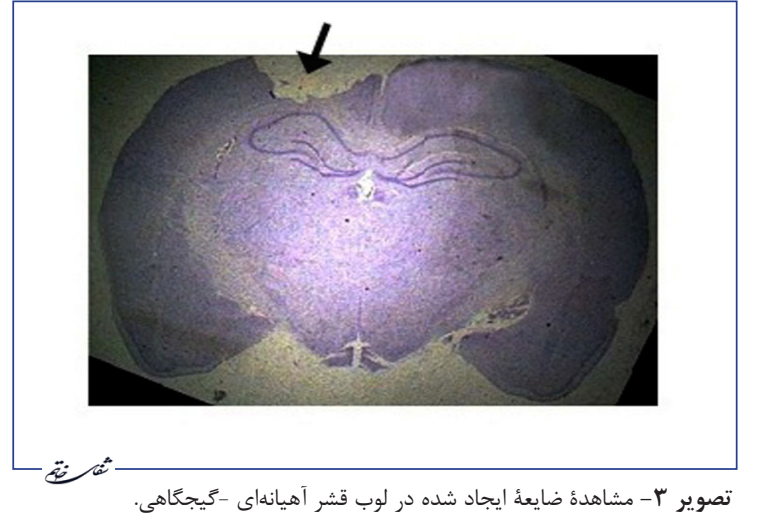

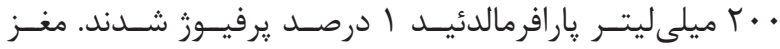

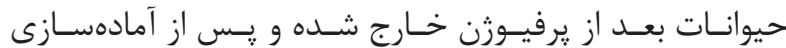

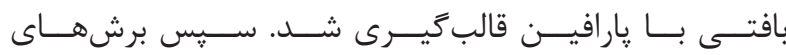

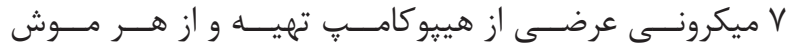

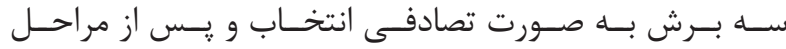

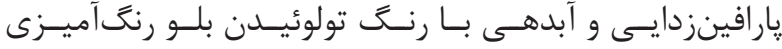

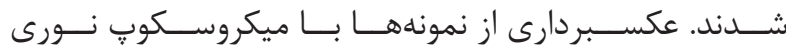

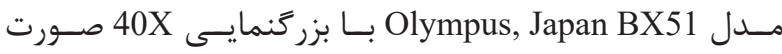

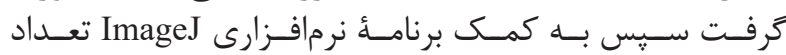

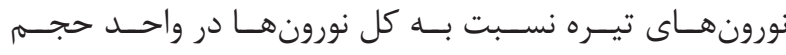

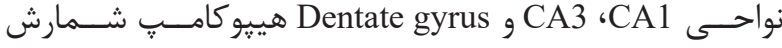

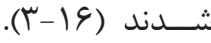

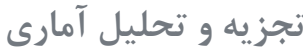

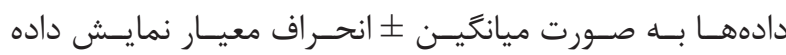

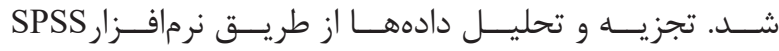

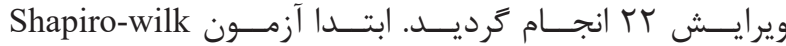

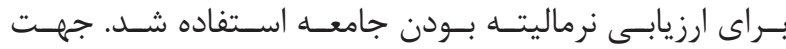

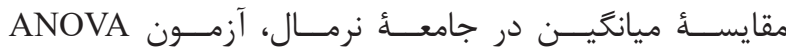

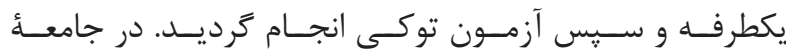

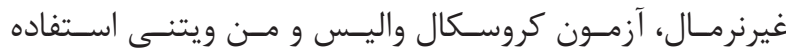

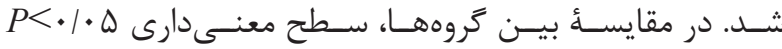
در نظــر كرفتـهـ شــد.

يافتهها

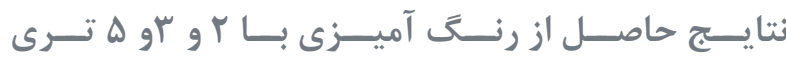

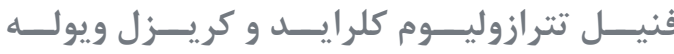

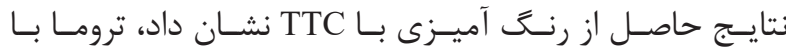

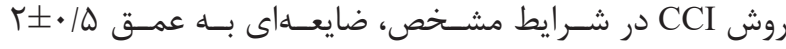

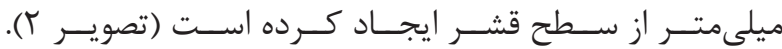

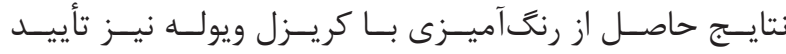

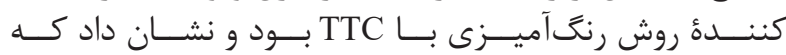

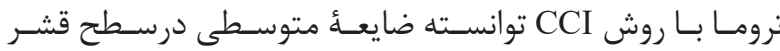

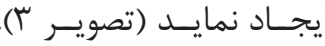

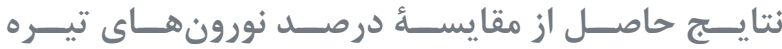
در نواحسى هيبو كامسِّ
روش پيش درمانى با لييو يلىساكاريد

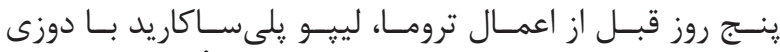

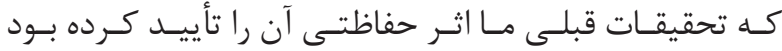

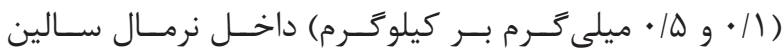

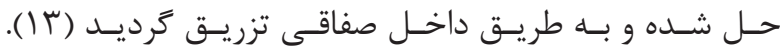

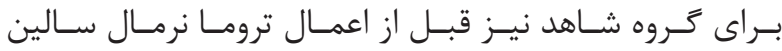

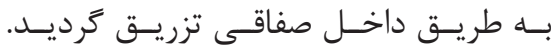
روش انجام مطالعات بافتى

\section{رنغَ آميزى با ب و ّاو ه ترى فنيل تترازوليوم كلرايد و

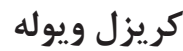

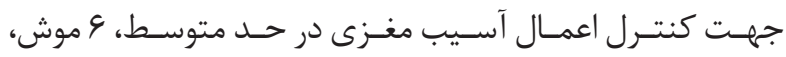

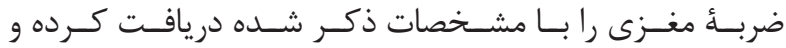

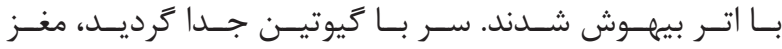

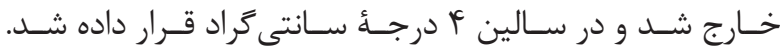

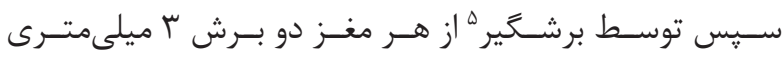

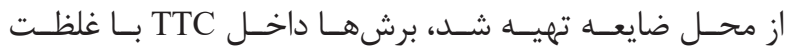

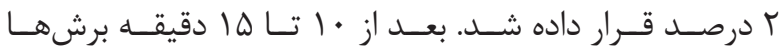

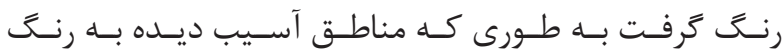

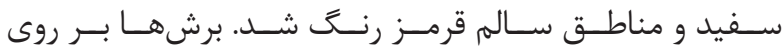

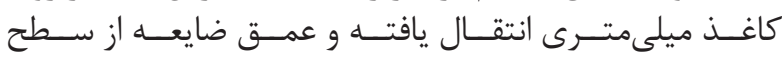

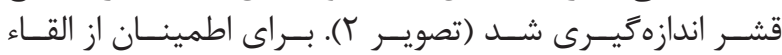

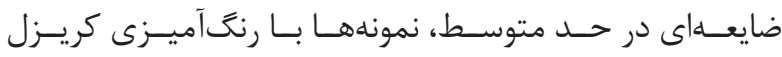

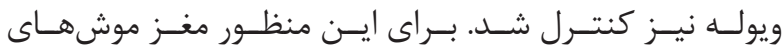

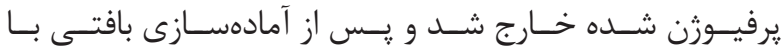

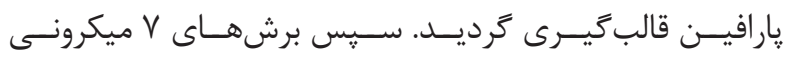

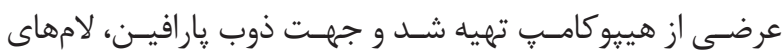

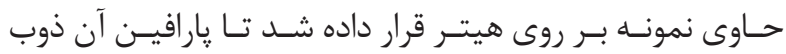

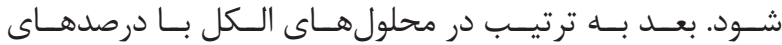

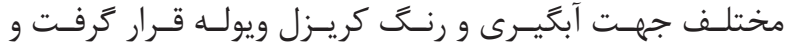

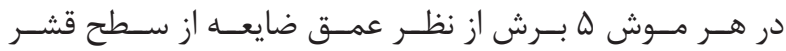

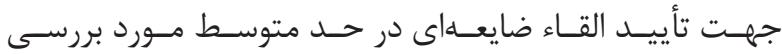

قـرار كرفت (تصويـر ساء). رنغ آميزى با تولوئيدن بلو

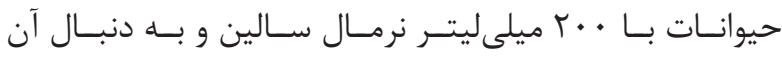

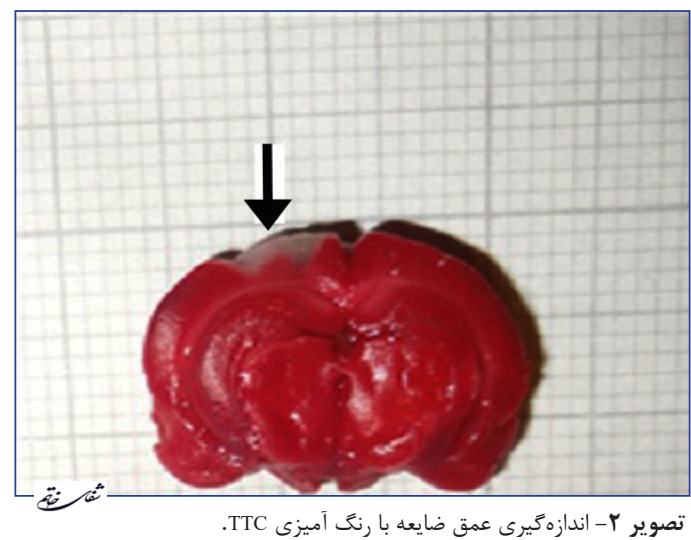




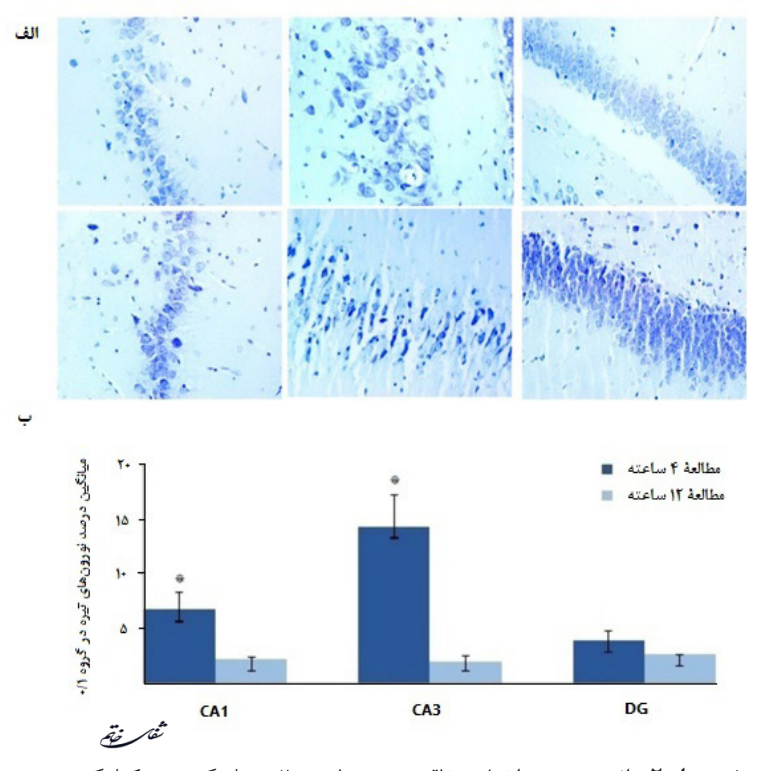

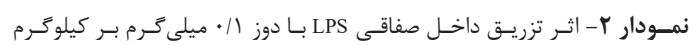

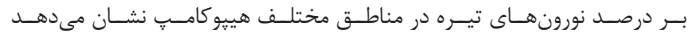

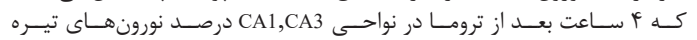

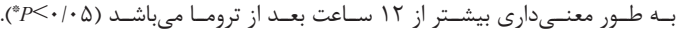

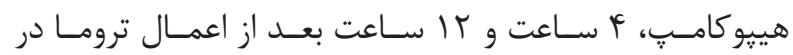

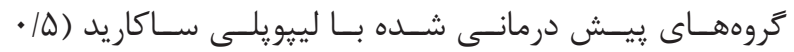

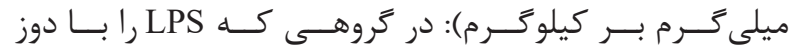

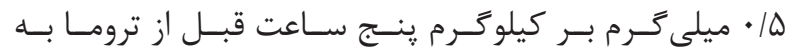

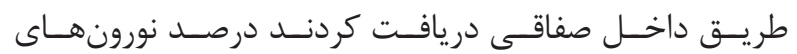

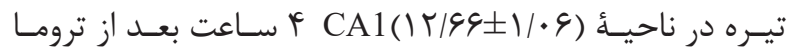

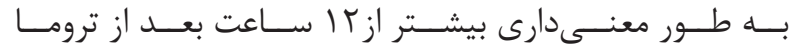

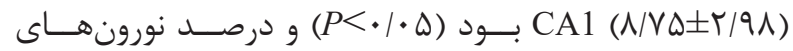

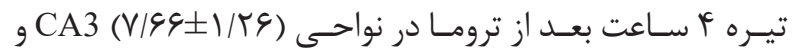

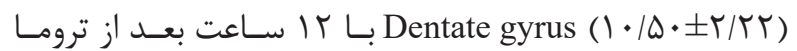

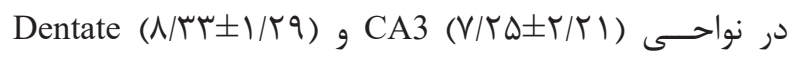

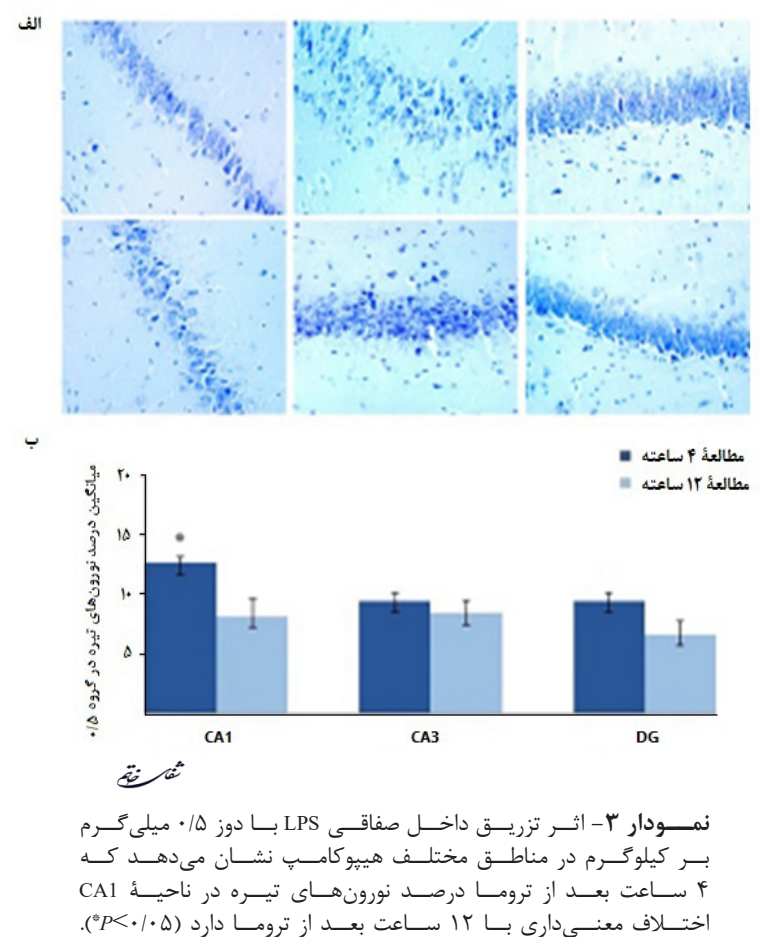

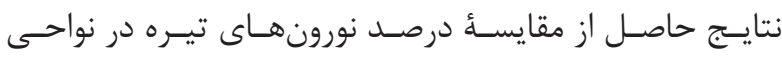

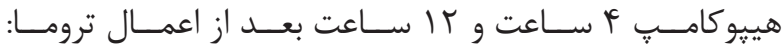

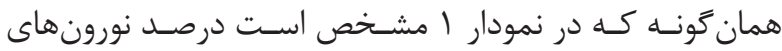

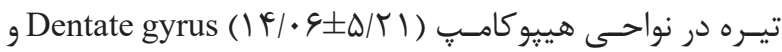

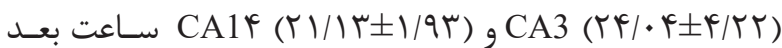

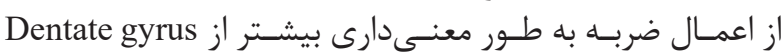
$(\mid r / \cdot r \pm r / \cdot V), C A 3(\mid r / q q \pm \Delta / / 1),(q / \cdot F \pm r / r r)$

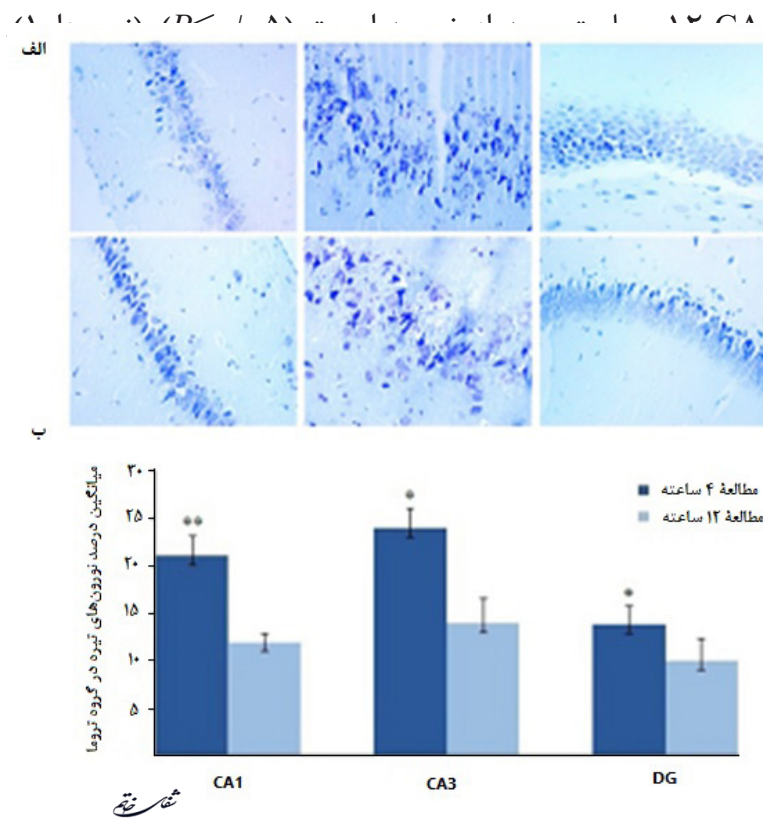

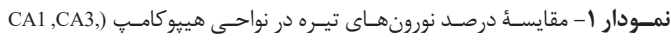
F Fentate gyrus

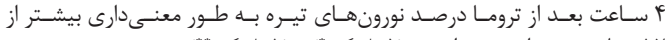

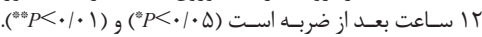

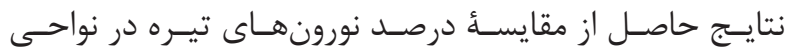

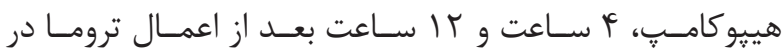

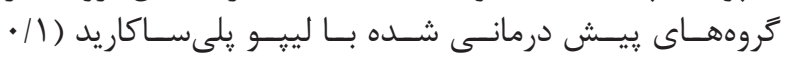

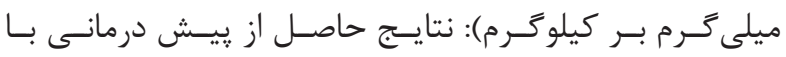

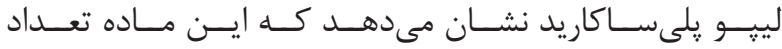

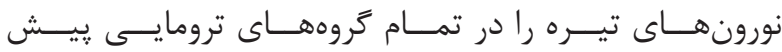

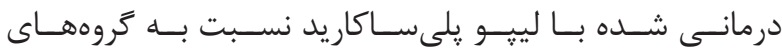

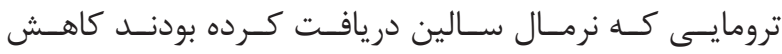

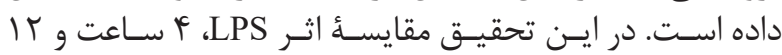

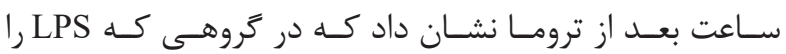

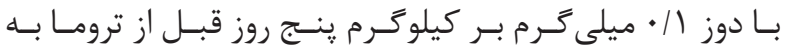

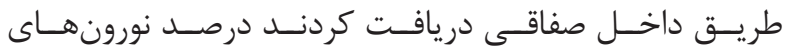
تيــره در نواحسى (CA3 ) و (

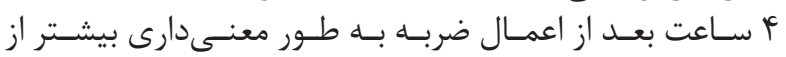

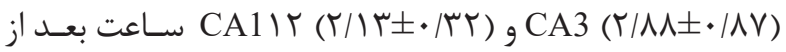

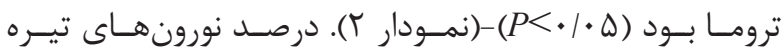
Dentate (r/AV

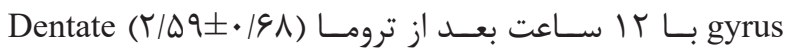
gyrus نتايـج حاصـل از مقايســُ درصـد نورونهــاى تيـره در نواحسى 


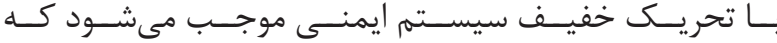

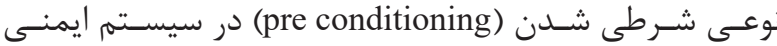

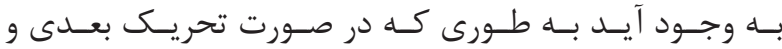

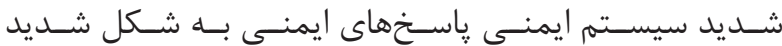

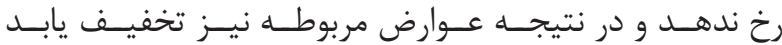

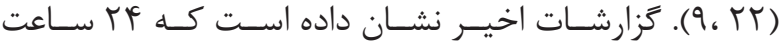

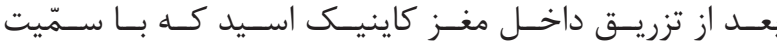

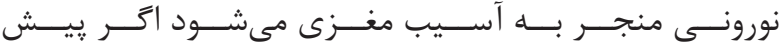

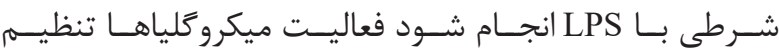

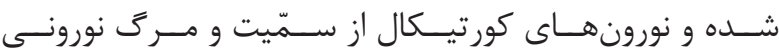

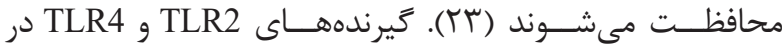

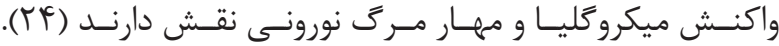

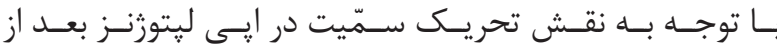

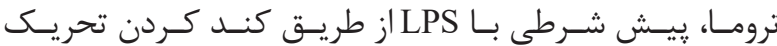

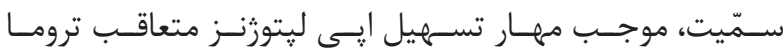

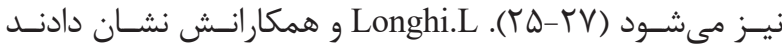

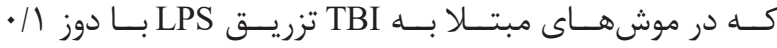

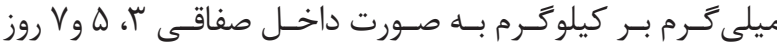

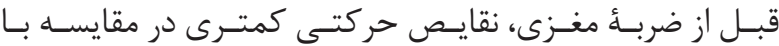

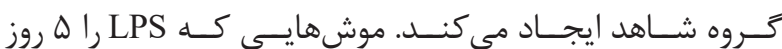

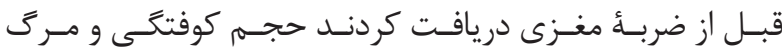

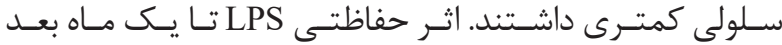

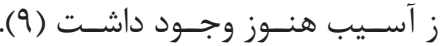

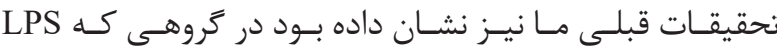

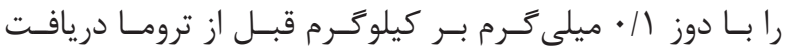

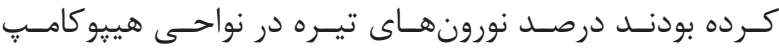

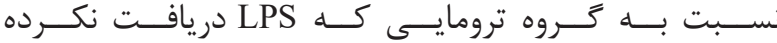

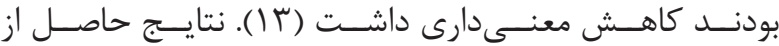

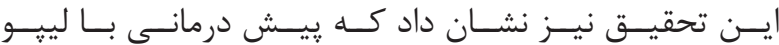

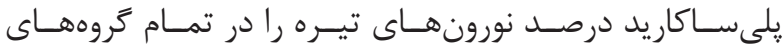

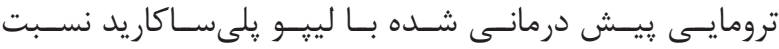

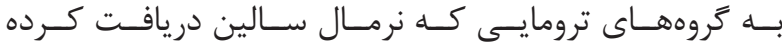

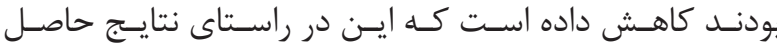

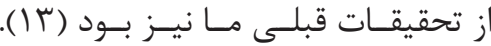

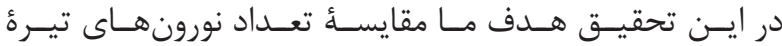
هيبوكامسـ

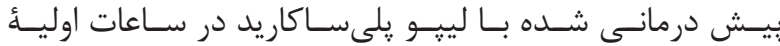

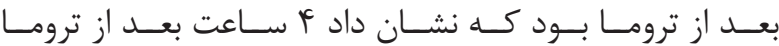

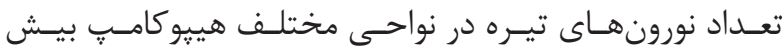

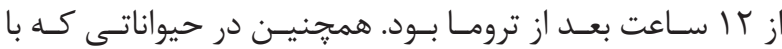
يـPS

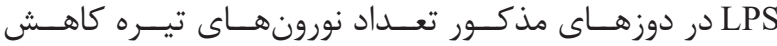

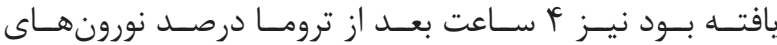

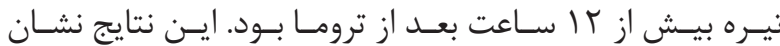

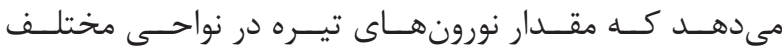

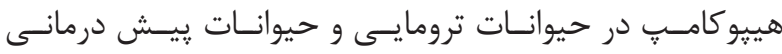

gyrus

بحث و نتيجه كَيرى

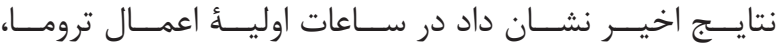

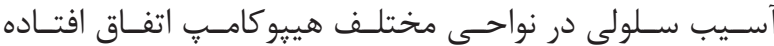

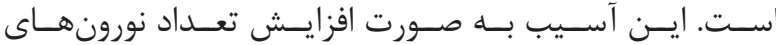

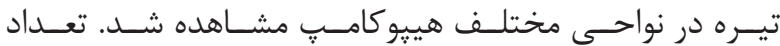

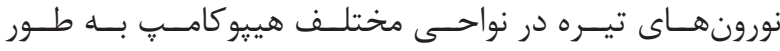

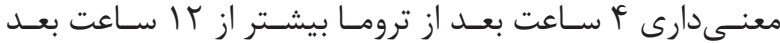

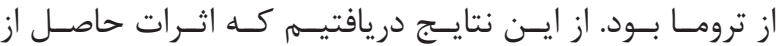

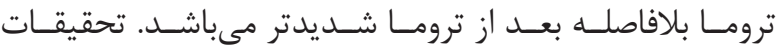

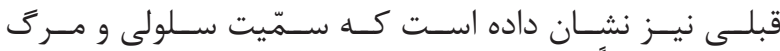

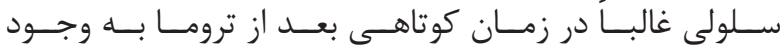

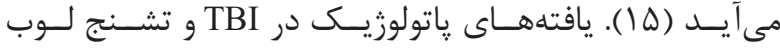

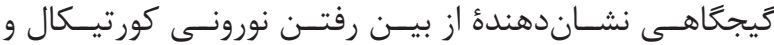

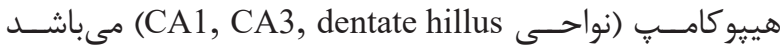

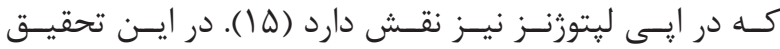

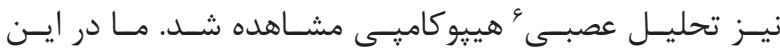

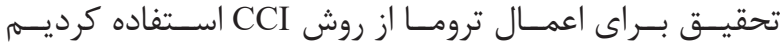

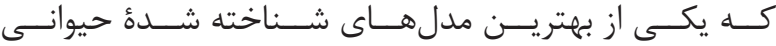

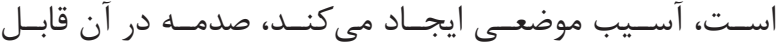

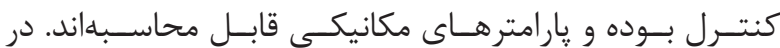

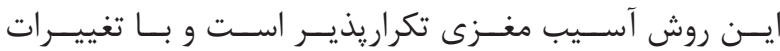

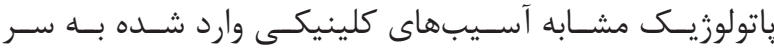

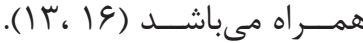

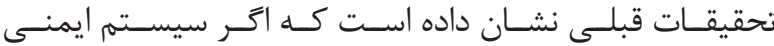

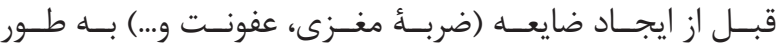

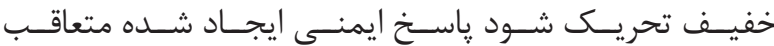

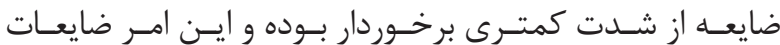

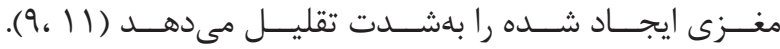

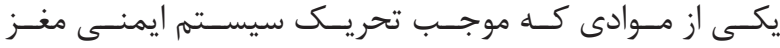

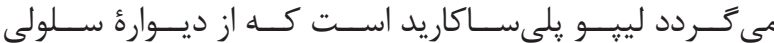

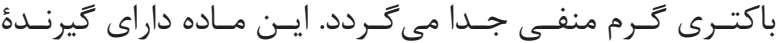

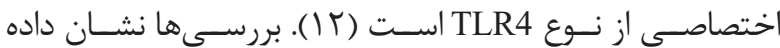

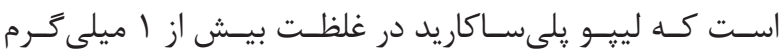

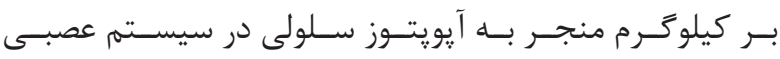

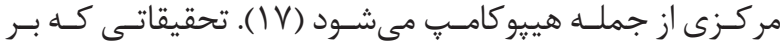

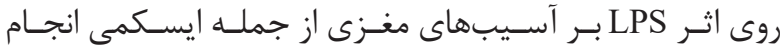

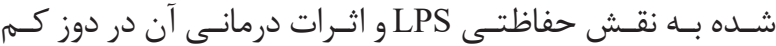

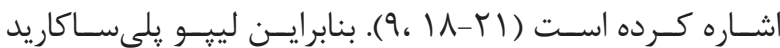

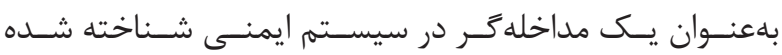

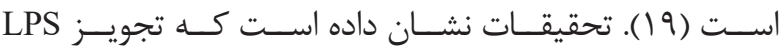

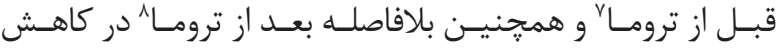

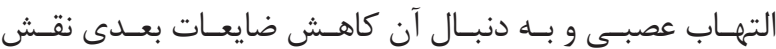

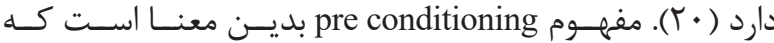

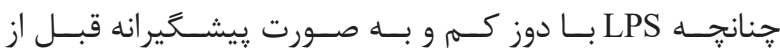

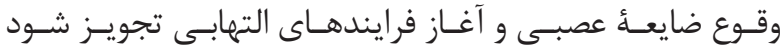

${ }^{6}$ Neurodegeneration

${ }^{7}$ Pre conditioning

${ }^{8}$ Post conditioning 


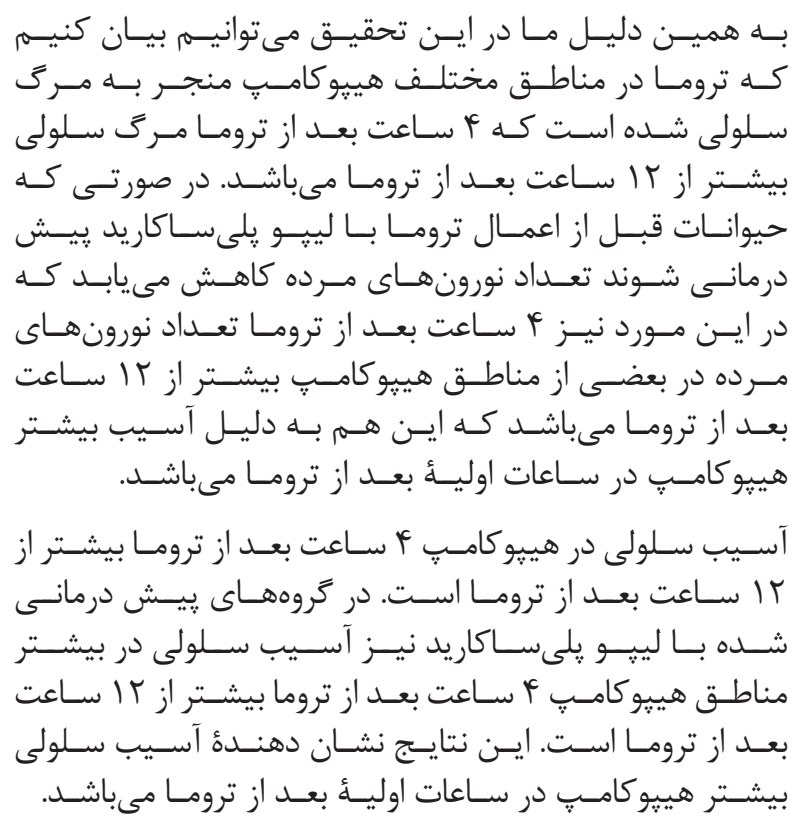

1. Leonardi M, Ustun TB. The global burden of epilepsy. Epilepsia. 2002; 43(6): 1-5.

2. Dudek FE, Staley KJ. The time course of acquired epilepsy: implications for therapeutic intervention to suppress epileptogenesis. Neurosci Lett. 2011; 497(3): 240-6.

3. Blumenfeld H. New strategies for preventing epileptogenesis: perspective and overview. Neurosci Lett. 2011; 497(3): 153-4.

4. Karimi M, Naseri M, Sayyah M, Kamalinejad M, Amiri S. Evaluation of anticonvulsive effect of methanol extract of ferula asafoetida gum in mice. Shefaye Khatam. 2014; 2(1): 46-50

5. Eftekhar B, Sahraian MA, Nouralishahi B, Khaji A, Vahabi Z, Ghodsi M, et al. Prognostic factors in the persistence of posttraumatic epilepsy after penetrating head injuries sustained in war: clinical article. J Neurosurg. 2009; 110(2): 319-26.

6. Ravizza T, Balosso S, Vezzani A. Inflammation and prevention of epileptogenesis. Neurosci Lett. 2011; 497(3): 223-30.

7. Granata T, Cross H, Theodore W, Avanzini G. Immune-mediated epilepsies. Epilepsia. 2011; 52(3): 5-11.

8. Aarli JA. Epilepsy and the immune system. Arch Neurol. 2000; 57(12): 1689-92.

9. Longhi L, Gesuete R, Perego C, Ortolano F, Sacchi $\mathrm{N}$, Villa $\mathrm{P}$, et al. Long-lasting protection in brain

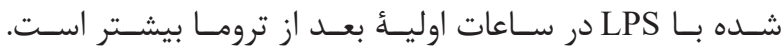

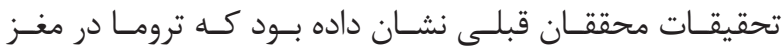

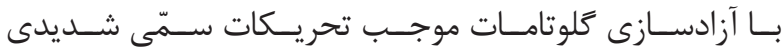

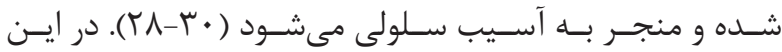

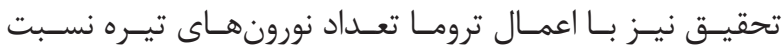

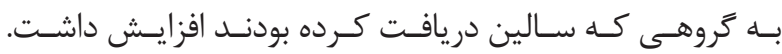

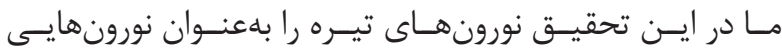

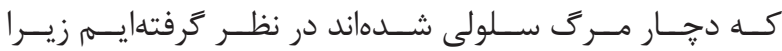

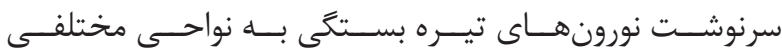

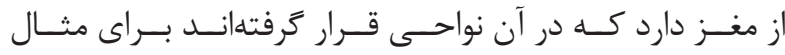

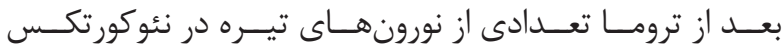

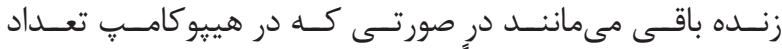

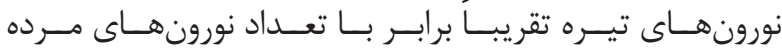

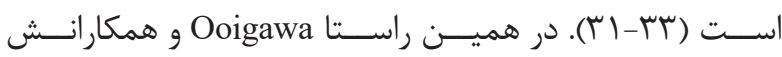

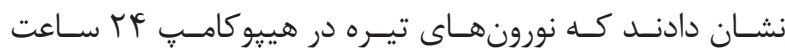

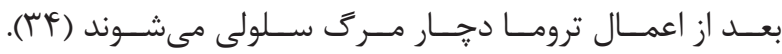

منابع

trauma by endotoxin preconditioning. J Cereb Blood Flow Metab. 2011; 31(9): 1919-29.

10. Lin HY, Huang CC, Chang KF. Lipopolysaccharide preconditioning reduces neuroinflammation against hypoxic ischemia and provides long-term outcome of neuroprotection in neonatal rat. Pediatr Res. 2009; 66(3): 254-9.

11. Mirrione MM, Konomos DK, Gravanis I, Dewey SL, Aguzzi A, Heppner FL, et al. Microglial ablation and lipopolysaccharide preconditioning affects pilocarpine-induced seizures in mice. Neurobiol Dis. 2010; 39(1): 85-97.

12. Marsh BJ, Williams-Karnesky RL, StenzelPoore MP. Toll-like receptor signaling in endogenous neuroprotection and stroke. Neuroscience. 2009; 158(3): 1007-20.

13. Eslami M, Sayyah M, Soleimani M, Alizadeh L, Hadjighassem M. Lipopolysaccharide preconditioning prevents acceleration of kindling epileptogenesis induced by traumatic brain injury. J Neuroimmunol. 2015; 289: 143-51.

14. Hu K, Li S-y, Xiao B, Bi F-f, Lu X-q, Wu $\mathrm{X}-\mathrm{m}$. Protective effects of quercetin against status epilepticus induced hippocampal neuronal injury in rats: involvement of X-linked inhibitor of apoptosis protein. Acta Neurol Belg. 2011; 111(3): 205-12.

15. Nourzad Z, Ghadiri T, Modarres Mousavi M, Karimzadeh F, Eshaghabadi A, Hosseini Ravandi H, et al. Neuroprotective effects of concomitant use of erythropoietin and progesterone in traumatic brain 


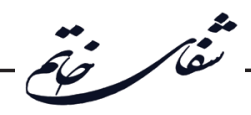

injury. Shefaye Khatam. 2014: 2(2): 1-12.

16. Alizadeh L, Gorizan A, Akbari Dana M, Ghaemi A. Immunotherapy of glioblastoma multiforme tumors: from basic to clinical trial studies. Shefaye Khatam. 2015; 3(2): 77-84.

17. Yu JT, Lee CH, Yoo K-Y, Choi JH, Li H, Park OK, et al. Maintenance of anti-inflammatory cytokines and reduction of glial activation in the ischemic hippocampal $\mathrm{CA} 1$ region preconditioned with lipopolysaccharide. $\mathrm{J}$ Neurol Sci. 2010; 296(1-2): 69-78.

18. Hassan H, Grecksch G, Rüthrich H, Krug M. Effects of nicardipine, an antagonist of L-type voltagedependent calcium channels, on kindling development, kindling-induced learning deficits and hippocampal potentiation phenomena. Neuropharmacology. 1999; 38(12): 1841-50.

19. Rosenzweig HL, Minami M, Lessov NS, Coste SC, Stevens SL, Henshall DC, et al. Endotoxin preconditioning protects against the cytotoxic effects of TNF $\alpha$ after stroke: a novel role for TNF $\alpha$ in LPSischemic tolerance. J Cereb Blood Flow Metab. 2007; 27(10): 1663-74.

20. Bingham D, John CM, Panter SS, Jarvis GA. Post-injury treatment with lipopolysaccharide or lipooligosaccharide protects rat neuronal and glial cell cultures. Brain Res Bull. 2011; 85(6): 403-9.

21. Pitkänen A, Bolkvadze T, Immonen R. Antiepileptogenesis in rodent post-traumatic epilepsy models. Neurosci Lett. 2011; 497(3): 163-71.

22. Löscher W, Brandt C. Prevention or modification of epileptogenesis after brain insults: experimental approaches and translational research. Pharmacol Rev. 2010; 62(4): 668-700.

23. Larochelle A, Bellavance MA, Rivest S. Role of adaptor protein MyD88 in TLR-mediated preconditioning and neuroprotection after acute excitotoxicity. Brain Behav Immun. 2015; 46: 221-31.

24. Ren Z, Iliff JJ, Yang L, Yang J, Chen X, Chen MJ, et al. 'Hit \& Run'model of closed-skull traumatic brain injury (TBI) reveals complex patterns of posttraumatic AQP4 dysregulation. J Cereb Blood Flow Metab. 2013; 33(6): 834-45.
25. Hunt RF, Boychuk JA, Smith BN. Neural circuit mechanisms of post-traumatic epilepsy. Front Cell Neurosci. 2013; 7: 89. doi: 10.3389/fncel.2013.00089.

26. Goodrich GS, Kabakov AY, Hameed MQ, Dhamne SC, Rosenberg PA, Rotenberg A. Ceftriaxone treatment after traumatic brain injury restores expression of the glutamate transporter, GLT-1, reduces regional gliosis, and reduces post-traumatic seizures in the rat. $\mathrm{J}$ Neurotrauma. 2013; 30(16): 1434-41.

27. Vandresen-Filho S, Hoeller AA, Herculano BA, Duzzioni M, Duarte FS, Piermartiri TC, et al. NMDA preconditioning attenuates cortical and hippocampal seizures induced by intracerebroventricular quinolinic acid infusion. Neurotox Res. 2013; 24(1): 55-62.

28. Meldrum BS. Excitotoxicity and selective neuronal loss in epilepsy. Brain Pathology. 1993; 3(4): 405-12.

29. Parsons MP, Raymond LA. Extrasynaptic NMDA receptor involvement in central nervous system disorders. Neuron. 2014; 82(2): 279-93.

30. Jayaram B, Khan RS, Kastin AJ, Hsuchou H, Wu $\mathrm{X}$, Pan W. Protective role of astrocytic leptin signaling against excitotoxicity. J Mol Neurosci. 2013; 49(3): 523-30.

31. Cortez SC, McIntosh T, Noble L. Experimental fluid percussion brain injury: vascular disruption and neuronal and glial alterations. Brain Res. 1989; 482(2): 271-82.

32. Lowenstein DH, Thomas MJ, Smith DH, McIntosh TK. Selective vulnerability of dentate hilar neurons following traumatic brain injury: a potential mechanistic link between head trauma and disorders of the hippocampus. J Neurosci. 1992; 12(12): 4846-53.

33. Nawashiro H, Shima K, Chigasaki H. Selective vulnerability of hippocampal CA3 neurons to hypoxia after mild concussion in the rat. Neurol Res. 1995; 17(6): 455-60.

34. Ooigawa $H$, Nawashiro $H$, Fukui S, Otani $N$, Osumi A, Toyooka T, et al. The fate of Nissl-stained dark neurons following traumatic brain injury in rats: difference between neocortex and hippocampus regarding survival rate. Acta Neuropathol. 2006; 112(4): 471-81. 